\title{
Correlation between the vitamin D levels and asthma attacks in children: Evaluation of the effects of combination therapy of atomization inhalation of budesonide, albuterol and vitamin D supplementation on asthmatic patients
}

\author{
QINHONG KANG, XIAOYING ZHANG, SHANJUAN LIU and FANG HUANG
}

Department of Pharmacy, Jingzhou Maternal and Child Health-Care Hospital, Jingzhou, Hubei 434020, P.R. China

Received April 20,2017; Accepted September 20, 2017

DOI: $10.3892 /$ etm.2017.5436

\begin{abstract}
The aim of the present study was to analyze the correlation between the vitamin D (VitD) levels and asthma attack in children, and to evaluate the effects of combination therapy of atomization inhalation of budesonide, albuterol and VitD supplementation on asthmatic children. The total sample size comprised of 96 children with asthma from the time period between May 2015 to April 2016. At the same time, 96 healthy children were also selected from the physical examination center for comparison study. The levels of serum VitD in both groups were detected by the enzyme-linked immunosorbent assay (ELISA). Pulmonary function index that includes the measurement of FEV1, FVC, FEV1/FVC, MEF25 and MEF50 were performed to analyze the results. The patients in the control group were treated with only the budesonide therapy and the patients in the observation group were treated with atomization inhalation of budesonide combined with salbutamol and VitD supplementation. After the treatment, the levels of inflammatory cell indicators (IL-2, IL-4, IL-6 and IFN- $\gamma$ ) and pulmonary function in the two groups were compared. The levels of serum VitD in the children with asthma were significantly lower than those in the normal children. The serum IgE level in children with asthma was significantly higher than that in the normal children $(\mathrm{P}<0.05)$. Pearson correlation coefficient analyses showed that VitD levels were not correlated with FEV1, FVC and FEV1/FVC levels $(\mathrm{P}>0.05)$, but was positively correlated with MEF25 and MEF50 $(\mathrm{P}<0.05)$. After the treatment, the levels of IL-2 and IFN- $\gamma$ in the observation group were significantly higher and levels of IL-4 and IL- 6 were significantly lower than those in the control group $(\mathrm{P}<0.05)$. The pulmonary function (FEV1, FVC, FEV1/FVC, MEF25 and MEF50) of the observation group was better than that of the control group $(\mathrm{P}<0.05)$.
\end{abstract}

Correspondence to: Dr Qinhong Kang, Department of Pharmacy, Jingzhou Maternal and Child Health-Care Hospital, 203 Jingzhou Middle Road, Jingzhou, Hubei 434020, P.R. China

E-mail: fkk4bc@163.com

Key words: vitamin D, children, asthma, budesonide, albuterol
The serum VitD levels of children with asthma were closely related to the acute asthmatic attacks. The lower the levels of serum VitD further leads to higher possibility of asthmatic attacks. Atomization inhalation of budesonide combined with albuterol and VitD supplementation can significantly improve the inflammatory response of the children with asthma.

\section{Introduction}

Industrial pollution and traffic emissions have increased the smog phenomenon. This coupled with vulnerable airway barrier of the under-age children further increase the incidence of asthma in children (1). Asthma in children has become a public health problem that has attracted the global concern regarding the disease. Various epidemiological studies have shown that the global prevalence of asthma in children ranges from 3 to $29 \%$ (2). The occurrence of asthma in children can be caused by many factors, including environmental, gender, genetic, ethnic factors and socioeconomic status (3). In recent years, studies have shown that vitamin D (VitD) levels and the incidence of asthma were closely related. Relevant studies have suggested that the VitD may be involved in the development of asthma or the lack of lung compliance will lead to immune dysfunction, thereby triggering the factors for asthma in children (4).

There has been a growing interest in the potential role of VitD in asthma management, because it might help to reduce upper respiratory infections (such as the common cold) that can lead to exacerbations of asthma. Several clinical trials have tested whether taking VitD as a supplement has an effect on asthma attacks, symptoms and lung function in children and adults with asthma. VitD supplementation can regulate the body's immune function, reduce inflammation and initiate repair functions in the body (5). It has been reported that the VitD has a protective effect on the respiratory tract (6). In the present study, VitD levels in normal children and children with asthma were detected and the correlation between the VitD levels and asthma attacks were investigated. Atomization inhalation of budesonide combined with albuterol and VitD supplement was used to treat asthma. This study provided a theoretical basis for effective prevention and treatment of asthma in children. 
Table I. Comparison of the general information between asthmatic groups.

\begin{tabular}{lcccr}
\hline Items & $\begin{array}{c}\text { Control group } \\
(\mathrm{n}=48)\end{array}$ & $\begin{array}{c}\text { Observation group } \\
(\mathrm{n}=48)\end{array}$ & $\mathrm{t} / \chi^{2}$ & P-value \\
\hline Sex (male/female) & $21 / 27$ & $25 / 23$ & 0.376 & 0.539 \\
Age (years) & $0-13$ & $0-14$ & & \\
Mean age (years) & $6.48 \pm 1.27$ & $6.56 \pm 1.38$ & 0.296 & 0.768 \\
Weight (kg) & $16.73 \pm 3.24$ & $17.07 \pm 3.33$ & 0.507 & 0.613 \\
History of allergies (n, \%) & $31(64.58)$ & $33(68.75)$ & 0.001 & 0.998 \\
Combined with mycoplasma pneumoniae infection (n, \%) & $16(33.33)$ & $18(37.50)$ & 0.047 & 0.827 \\
Eosinophil count in peripheral blood & $0.47 \pm 0.26$ & $0.45 \pm 0.24$ & 0.392 & 0.696 \\
Childhood Asthma Control Test Score (C-ACT) & $16.26 \pm 1.25$ & $16.14 \pm 1.34$ & 0.454 & 0.651 \\
\hline
\end{tabular}

\section{Materials and methods}

General information. The total sample size was comprised of 96 children with diagnosis of asthma. The data were collected from the time period of May 2015 to April 2016. At the same time, a total of 96 healthy children were also selected from the physical examination center. The study was approved by the Ethics Committee of Jingzhou Maternal and Child Health-Care Hospital and informed consents were signed by the guardians.

The inclusion criteria for the study was as followed: i) patients who meet the asthma diagnostic criteria established by Chinese Medical Association were included in the samples; ii) age between 0 and 14 years were the only children that were included in the samples; iii) the patients presenting with clinical manifestations of cough, chest tightness, lung wheezing, night and/or early morning onset or exacerbate; and iv) the parents had signed informed consent. The exclusion criteria that were taken for the study was as follows: i) the children without history of recurrent respiratory tract infections and recent history of wheezing were excluded from the sample size; ii) those children that were allergic to the drugs which were used in this study were also excluded; and iii) those children who were on hormonal drugs during the previous 30 days.

The asthmatic patients were further randomly subdivided into two groups i.e. observation group and control group based on the treatment protocol. Each group comprised of 48 cases. The patients in the control group were treated with the only budesonide therapy. The patients in the observation group were treated with atomization inhalation of budesonide combined with salbutamol and VitD supplementation. The general information of the both groups are given in Table I.

Determination of VitD levels and IgE content. The venous blood of $3 \mathrm{ml}$ was extracted from the all samples including the healthy subjects. After the centrifugation at the rate of $3000 \mathrm{r} / \mathrm{min}$ for $20 \mathrm{~min}$, the separated serum was placed in an EP tube and stored at $-80^{\circ} \mathrm{C}$. The levels of serum VitD were determined by ELISA. The serum VitD levels were determined according to the instructions of $25(\mathrm{OH}) \mathrm{D}_{3}$ kit (R\&D Systems, Inc., Minneapolis, MN, USA). After adding samples, incubation, washing, enzyme addition, again incubation and washing, the color development and the stop
Table II. Comparison of VitD and IgE levels in both groups of children.

\begin{tabular}{lccc}
\hline Groups & Cases & $\begin{array}{c}\text { VitD } \\
(\mathrm{ng} / \mathrm{ml})\end{array}$ & $\begin{array}{c}\text { IgE } \\
(\mathrm{IU} / \mathrm{ml})\end{array}$ \\
\hline Children with asthma & 96 & $18.89 \pm 3.63$ & $375.13 \pm 64.34$ \\
Normal children & 96 & $32.74 \pm 3.67$ & $286.79 \pm 54.45$ \\
t-value & & 26.289 & 10.269 \\
P-value & & $<0.001$ & $<0.001$ \\
\hline
\end{tabular}

Table III. Correlation between VitD levels, IgE and pulmonary function.

\begin{tabular}{lcc}
\hline Items & Correlation coefficient & P-value \\
\hline FEV1 & -0.568 & 0.103 \\
FVC & 0.601 & 0.214 \\
FEV1/FVC & 0.345 & 0.317 \\
MEF25 & 0.679 & 0.005 \\
MEF50 & 0.457 & 0.016 \\
IgE & -0.568 & 0.024
\end{tabular}

solution was added within $15 \mathrm{~min}$. The OD of each well was measured by a microplate reader (450 $\mathrm{nm}$ wavelength) and the VitD level was calculated. The total serum IgE level was measured by immunoturbidimetry according to the instructions of IgE kit (Weifang Kanghua Biotechnology Co., Ltd., Weifang, China).

Treatment of asthmatic children. The children in the control group were given only budesonide nasal spray (AstraZeneca AB, Södertälje, Sweden; National medical license number: J20040082) inhalation therapy. Children that were younger than 6 years were given a dose of $100 \mu \mathrm{g}$ and children aged 6 years or above were given a dose of $200 \mu \mathrm{g}$, twice a day. Besides budesonide treatment, the patients in the observation group were given salbutamol aerosol inhalation solution 

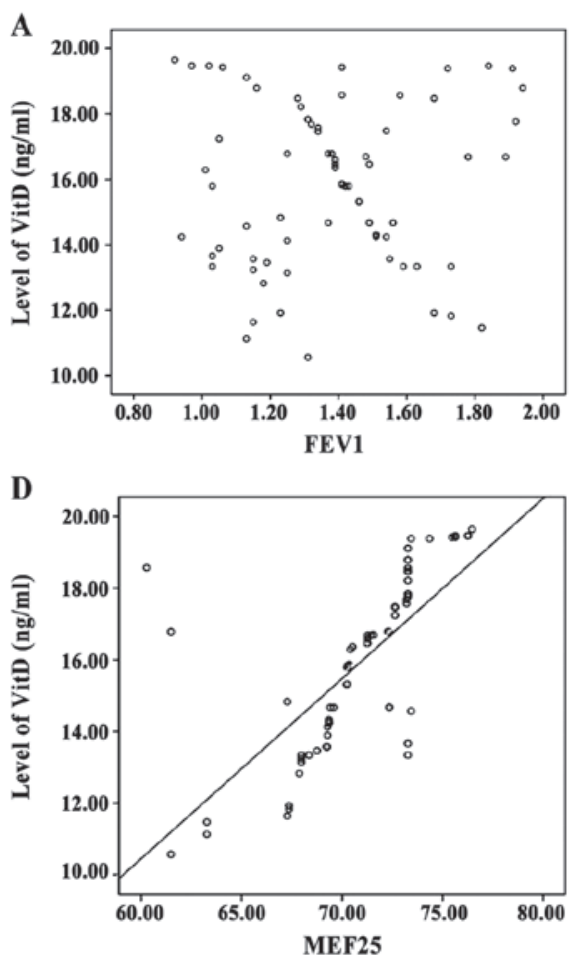
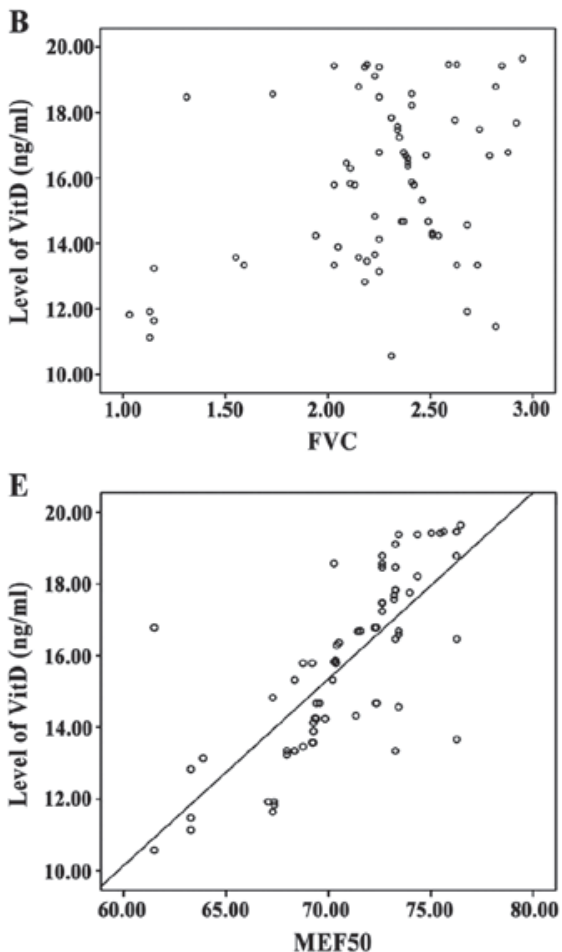

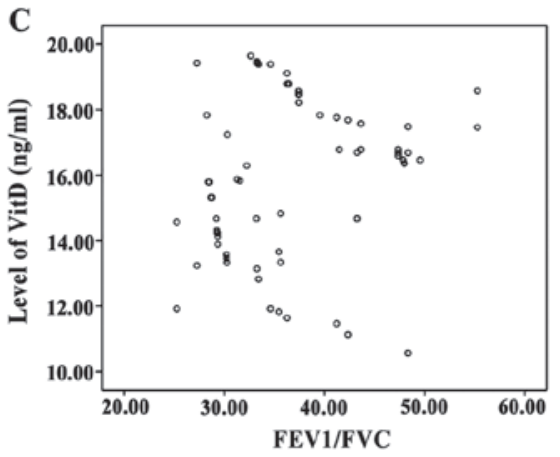

F

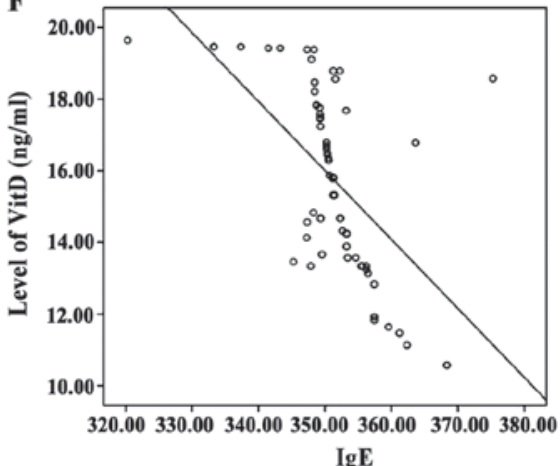

Figure 1. Scattergraph of vitamin D (VitD) levels and several indicators. (A) FEV1 levels P>0.05; (B) FVC levels P>0.05; (C) FEV1/FVC levels P>0.05; (D) MEF25 levels $\mathrm{P}<0.05$; (E) MEF50 levels $\mathrm{P}<0.05$; (F) IgE levels $\mathrm{P}<0.05$.

(Shanghai Xinyi Jinzhu Pharmaceutical Co., Ltd., Shanghai, China; National medical license number: H19990233) and with further supplementation of vitamin D (Qingdao Shuangjing Pharmaceutical Co., Ltd., Qingdao, China; National medical license number: H20113033). The dosage of salbutamol therapy was as followed: children who were under the age of 6 were given $100 \mu \mathrm{g}$, twice a day and the dosage was doubled in cases of severe sign and symptoms. Children who were aged 6 years or above were given a dose of $200 \mu \mathrm{g}$, twice a day, the drug was given once every $6 \mathrm{~h}$ for severe symptom, $200 \mu \mathrm{g}$ each time. The dosage of VitD was as followed: children under the age of 6 were given 200 IU per day and children over the age of 6 were given $400 \mathrm{IU}$ per day.

Detection of the indicators. Pulmonary function was detected by children's pulmonary function monitor (Master Screen Paed, CareFusion Corp., Höchberg, Germany). While testing, the respiratory tract was kept unobstructed. Ring finger and fifth finger was used to hold up the jaw of the children to put the mask, so that it covered both the nose and mouth. The indicators which were used in the study were: forced expiratory volume 1 (FEV1), forced vital capacity (FVC), FEV1/FVC ratio, maximum expiratory flow at 25\% (MEF25) and maximum expiratory flow at 50\% (MEF50). These tests were measured under quiet breathing.

After 3 months of treatment, again the venous blood samples were taken at early morning ( $8 \mathrm{~h}$ fasting), and the serum was separated by centrifugation. IL-2, IL-4, IL-6 and IFN- $\gamma$ levels were determined by ELISA in strict accordance with the instructions (Aikang Biology Co., Ltd., Shaoxing, China). Concentrations of IL-2, IL-4, IL-6 and IFN- $\gamma$ were calculated by reading the OD values using a microplate reader (450 nm wavelength). All patients were followed up for 6 months. The asthma control rate, reoccurrence rate and re-hospitalization rate were statistically analyzed.

Follow-up. The control rate, reoccurrence rate and re-hospitalization rate were compared between the groups 6 months after the discharge. The standard criteria which were set for both groups are as follows: Asthma control rate, no recurrence of asthma within half a year. Recurrence rate, at least one acute attack within six months. Re-hospitalization rate, hospitalization with six months due to asthma recurrence.

Statistical analysis. Data were processed using SPSS software version 19.0 (SPSS, Inc., Chicago, IL, USA). Measurement data were expressed as mean \pm SD and were analyzed by the $\mathrm{t}$-test. The count data were expressed in terms of rate and were analyzed by the $\chi^{2}$. Pearson correlation coefficient analysis was used to analyze the correlation between VitD levels and pulmonary function, IgE. $\mathrm{P}<0.05$ was considered to be statistically significant.

\section{Results}

Comparison of VitD and IgE levels in both groups of children. The levels of serum VitD in children with asthma were significantly lower than those in the normal children. The serum $\operatorname{IgE}$ levels in children with asthma were significantly higher than those in normal children, and this was statistically significant at $\mathrm{P}<0.05$ (Table II).

Correlation between VitD levels, IgE and pulmonary function. Pearson correlation coefficient analysis showed that 
Table IV. Comparison of pulmonary function in asthmatic groups of children.

\begin{tabular}{lcccccc}
\hline Groups & N (cases) & FVC (L) & FEV1 (L) & FEV1/FVC $(\%)$ & MEF25 (\%) & MEF50 (\%) \\
\hline Control group & 48 & $2.35 \pm 0.73$ & $1.19 \pm 0.37$ & $41.35 \pm 8.34$ & $65.14 \pm 7.48$ & $65.34 \pm 3.15$ \\
Observation group & 48 & $2.69 \pm 0.87$ & $1.46 \pm 0.42$ & $49.53 \pm 7.76$ & $78.28 \pm 7.24$ & $79.48 \pm 3.38$ \\
t-value & & 1.933 & 3.342 & 4.957 & 8.745 & 21.203 \\
P-value & & 0.041 & 0.001 & $<0.001$ & $<0.001$ & $<0.001$ \\
\hline
\end{tabular}

Table V. The comparison of cytokine indexes between the asthmatic groups after the treatment.

\begin{tabular}{lccccc}
\hline Groups & N (cases) & IL-2 $(\mu \mathrm{mol} /)$ & IL-4 $(\mu \mathrm{mol} /)$ & IL-6 $(\mu \mathrm{mol} / \mathrm{l})$ & IFN- $\gamma(\mu \mathrm{mol} /)$ \\
\hline Control group & 48 & $56.57 \pm 4.13$ & $24.45 \pm 3.54$ & $19.72 \pm 5.46$ & $18.57 \pm 6.34$ \\
Observation group & 48 & $51.36 \pm 4.38$ & $31.64 \pm 3.38$ & $23.48 \pm 5.57$ & $14.25 \pm 6.73$ \\
t-value & & 5.966 & 10.178 & 3.340 & 3.237 \\
P-value & $<0.001$ & $<0.001$ & 0.001 & 0.001 \\
\hline
\end{tabular}

Table VI. Comparison of therapeutic effect observed 6 months after discharge in the asthmatic groups.

\begin{tabular}{lcccc}
\hline Groups & N (cases) & Asthma control rate & Recurrence rate & Re-hospitalization rate \\
\hline Control group & 48 & $44(91.67)$ & $4(8.33)$ & $3(6.25)$ \\
Observation group & 48 & $35(72.92)$ & $14(29.17)$ & $11(22.92)$ \\
$\chi^{2}$ & & 59.54 & 58.73 & 59.82 \\
P-value & & 0.032 & 0.018 & 0.042 \\
\hline
\end{tabular}

VitD levels were not significantly correlated with FEV1, FVC, FEV1/FVC, but positively correlated with MEF25 and MEF50. The analysis also revealed that VitD levels were negatively correlated with $\operatorname{IgE}$ and were statistically significant at $\mathrm{P}<0.05$ (Table III and Fig. 1).

Comparison of pulmonary function in the asthmatic groups of children. After 3 months of treatment, the FVC, FEV1, FEV1/ FVC, MEF25 and MEF50 levels in observation group were significantly higher than the control group with significance at $\mathrm{P}<0.05$ (Table IV).

The comparison of cytokine indexes between the asthmatic groups after the treatment. The level of IL-2 and IFN- $\gamma$ in the observation group were significantly higher than those in the control group $(\mathrm{P}<0.05)$. The level of IL-4 and IL-6 in the observation group was significantly lower than that in the control group with statistical significance at $\mathrm{P}<0.05$ (Table V).

Comparison of therapeutic effect observed 6 months after discharge in the asthmatic groups. The asthma recurrence rate and re-hospitalization rate were significantly lower in the observation group than those in the control group. The asthma control rate in the observation group was significantly higher than that in the control group with statistical significance at $\mathrm{P}<0.05$ (Table VI).

\section{Discussion}

Asthma is also known as bronchial asthma, basically it is a chronic airway inflammation with a very high incidence in children. Asthma symptoms in children are more significantly noticed at night or early morning. The symptom can be relieved by the appropriate treatment therapy (7). One of the main and common causes of asthma is the chronic inflammatory reactions of the respiratory tract. Airway inflammation occur in both the periods of acute exacerbation of asthma and remission periods of asthma. The stimulating and aggravating factors like activation of allergen, hyperventilation, excessive exercise, cold air and strong emotional changes can increase the symptoms of airway inflammation, thus, inducing acute asthmatic attacks. Thus, the key to reduce the asthmatic attacks is to control the airway inflammation $(8,9)$.

Vitamin D (VitD), a second type of steroid derivative. VitD can be converted into the 7-dehydrocholesterol from cholesterol and be converted to vitamin D3 by ultraviolet light. After its absorption into blood, VitD is finally converted into a highly active form $25(\mathrm{OH}) \mathrm{D}_{3}$. At present, $25(\mathrm{OH}) \mathrm{D}_{3}$ is most commonly used in the evaluation of VitD levels. The biological half-life of VitD is approximately 3 weeks $(10,11)$. VitD not only plays an important role in regulating bone metabolism and calcium homeostasis, but also has a key active role in the immune regulation system. The beneficial effects of VitD on protective immunity are due in part to its effects on the innate 
immune system. It is known that macrophages recognize lipopolysacharide LPS, a surrogate for bacterial infection, through toll-like receptors (TLR). Engagement of TLRs leads to a cascade of events that produce peptides with potent bacteriacidal activity such as cathelocidin and beta-defensin 4 . These peptides colocalize within phagosomes with injested bacteria where they disrupt bacterial cell membranes and have potent anti-microbacterial activity. VitD can also regulate the blood calcium levels and tissue and cell differentiation (12). Studies have found that the VitD levels and early transient wheezing are closely related to each other, and higher infant cord serum VitD levels can usually lead to lower incidence of wheezing (13). The present study found that VitD levels in the children with asthma were significantly lower than those in normal children and serum IgE levels in children with asthma were significantly higher than those in the normal children with significance at $\mathrm{P}<0.05$. The reason is that imbalance of Th1/Th2 cytokines also leads to asthma. VitD is involved in the inhibition of autoimmune disorders and low VitD level will reduce the inhibitory effect, which further leads to the overexpression of Th2-type cytokines and eventually increase the occurrence of asthma (14).

This study also found that there was no correlation between VitD levels and FEV1, FVC, FEV1/FVC (P>0.05) and positively correlated with MEF25 and MEF50 $(\mathrm{P}<0.05)$. The results of the study showed that the VitD levels were negatively correlated with IgE. Some studies have shown similar results and concluded that the FEV1, FVC, FEV1/ FVC parameters are not related to the asthma symptoms, but small airway parameters MEF25 and MEF50 are related to asthma symptoms and inflammatory response. VitD can also reduce the division and proliferation of smooth muscle cells that further leads to the decreased airflow and also reduces the small airway obstruction $(15,16)$. Thus, we hypothesized that low VitD levels can decrease the resistant ability of children's airway to infection, leading to the higher airway infection and induce asthmatic attacks. Therefore, VitD supplementation can be considered as adjuvant therapy for the prevention and treatment of asthma in children.

As a kind of glucocorticoid, budesonide can inhibit the synthesis and release of broncho-constrictor substances. These substances reduce the contractile response of smooth muscle, and further, significantly alleviate the acute onset of asthmatic attacks (17). As the excitant of $\beta 2$ receptor, salbutamol has strong effects on bronchial smooth muscles. Salbutamol can relax the bronchial smooth muscle, relieve bronchial spasm and reduce the permeability of the peripheral capillaries (18). This study found that the levels of IL-2 and IFN- $\gamma$ in the observation group were higher than those in the control group with significant values $(\mathrm{P}<0.05)$, and the levels of IL-4 and IL- 6 in the observation group were significantly lower than those in the control group. This is because the budesonide and salbutamol can bind to the cysteine receptors, and VitD can be combined with the two drugs to reduce the sensitivity of peripheral blood eosinophils. In addition, VitD in combination with the two drugs can participate in the regulation of Th1/Th2 cytokine. That will further regulate the alveolar macrophages, pulmonary epithelial cells and dendritic cells to inhibit the production of IL-4 and IL- 6 cells, reducing airway inflammation and promoting the secretion of IL-2 and IFN- $\gamma$ that prevents the reoccurrence of airway hyper-responsiveness (19).

Animal model revealed that, the VitD can reduce the secretion of chemokines from airway smooth muscle cells and reduce the pathological changes of airway remodeling (20). The present study also found similar results. The results revealed that the reoccurrence rate and re-hospitalization rate of the observation group were significantly lower than those of the control group $(\mathrm{P}<0.05)$, suggesting that high VitD levels could reduce the recurrence of asthma. In conclusion, VitD levels are closely related with the onset of asthma in children. Atomization inhalation of budesonide combined with albuterol and VitD supplementation can significantly improve children's pulmonary function and reduce airway inflammation, which will increase the asthma control rate. Future studies with larger sample are still need to further investigate the mechanism.

\section{References}

1. Pasić A, Tahirović $\mathrm{H}$ and Hadzibeganović M: Incidence of asthma in children in Tuzla Canton-Bosnia and Herzegovina. Coll Antropol 35: 299-303, 2011

2. Zhang YX, Liu Y, Xue Y, Yang LY, Song GD and Zhao L: Correlational study on atmospheric concentrations of fine particulate matter and children cough variant asthma. Eur Rev Med Pharmacol Sci 20: 2650-2654, 2016.

3. Yin GQ, Jiang WH, Wu PQ, He CH, Chen RS and Deng L: Clinical evaluation of sublingual administration of dust mite drops in the treatment of allergic asthma and allergic rhinitis of children. Eur Rev Med Pharmacol Sci 20: 4348-4353, 2016.

4. Turkeli A, Ayaz O, Uncu A, Ozhan B, Bas VN, Tufan AK, Yilmaz $\mathrm{O}$ and Yuksel H: Effects of vitamin D levels on asthma control and severity in pre-school children. Eur Rev Med Pharmacol Sci 20: 26-36, 2016.

5. Hoxha M, Zoto M, Deda L and Vyshka G: Vitamin D and its role as a protective factor in allergy. Int Sch Res Notices 2014: 951946, 2014.

6. Baïz N, Dargent-Molina P, Wark JD, Souberbielle JC and AnnesiMaesano I; EDEN Mother-Child Cohort Study Group: Cord serum 25-hydroxyvitamin D and risk of early childhood transient wheezing and atopic dermatitis. J Allergy Clin Immunol 133: 147-153, 2014.

7. Gong L, Xu L, Diao M, Guo F, Bian FF, Min J, Liu R and Zhang CL: Clinical effect of treating secondary asthma attacks of children Mycoplasma pneumoniae with combined therapy of montelukast and azithromycin. Eur Rev Med Pharmacol Sci 20: 5256-5260, 2016.

8. Agarwal R, Dhooria S, Aggarwal AN, Maturu VN, Sehgal IS, Muthu V, Prasad KT, Yenge LB, Singh N, Behera D, et al: Guidelines for diagnosis and management of bronchial asthma: Joint ICS/NCCP (I) recommendations. Lung India 32 (Suppl 1): S3-S42, 2015.

9. Yadav M and Mittal K: Effect of vitamin D supplementation on moderate to severe bronchial asthma. Indian J Pediatr 81: 650-654, 2014.

10. Institute of Medicine (US) Committee to Review Dietary Reference Intakes for Vitamin D and Calcium. Dietary reference intakes for calcium and vitamin D. Pediatrics 130: 1427, 2011.

11. Dong $\mathrm{CH}$, Gao QM, Wang ZM, Wang AM and Zhen P: Vitamin D supplementation for osteoporosis in older adults: Can we make it help better? Eur Rev Med Pharmacol Sci 20: 4612-4621, 2016.

12. Mangge H, Zelzer S, Meinitzer A, Stelzer I, Schnedl WJ, Weghuber D, Fuchs D, Postolache TT, Aigner E, Datz C, et al: $25 \mathrm{OH}-\mathrm{Vitamin} \mathrm{D} 3$ levels in obesity and metabolic syndromeunaltered in young and not correlated to carotid IMT in all ages. Curr Pharm Des 21: 2243-2249, 2015.

13. Einisman H, Reyes ML, Angulo J, Cerda J, López-Lastra M and Castro-Rodriguez JA: Vitamin D levels and vitamin D receptor gene polymorphisms in asthmatic children: A case-control study. Pediatr Allergy Immunol 26: 545-550, 2015. 
14. Tamašauskienė L, Gasiūnienė E, Lavinskienė S, Sakalauskas R and Šitkauskienè B: Evaluation of vitamin D levels in allergic and non-allergic asthma. Medicina (Kaunas) 51: 321-327, 2015.

15. Ahmadabadi F, Mirzarahimi M, Barak, Ahadi A and Alipour R: The relationship between serum vitamin D level and asthma severity in asthmatic children (aged 1-15 years) in Ardabil, 2012-13. Int J Res Med Sci 3: 239-243, 2015.

16. Bener A, Ehlayel MS, Bener HZ and Hamid Q: The impact of Vitamin D deficiency on asthma, allergic rhinitis and wheezing in children: An emerging public health problem. J Family Community Med 21: 154-161, 2014.

17. Lazarinis N, Jørgensen L, Ekström T, Bjermer L, Dahlén B, Pullerits T, Hedlin G, Carlsen KH and Larsson K: Combination of budesonide/formoterol on demand improves asthma control by reducing exercise-induced bronchoconstriction. Thorax 69: 130-136, 2014

18. Visser R, van der Palen J, de Jongh FH and Thio BJ: Reversibility after inhaling salbutamol in different body postures in asthmatic children: A pilot study. Respir Med 109: 459-462, 2015.
19. Das I, Saha K, Mukhopadhyay D, Roy S, Raychaudhuri G, Chatterjee $M$ and Mitra PK: Impact of iron deficiency anemia on cell-mediated and humoral immunity in children: A case control study. J Nat Sci Biol Med 5: 158-163, 2014.

20. Alyasin S, Momen T, Kashef S, Alipour A and Amin R: The relationship between serum 25 hydroxy vitamin d levels and asthma in children. Allergy Asthma Immunol Res 3: 251-255, 2011.

This work is licensed under a Creative Commons Attribution-NonCommercial-NoDerivatives 4.0 International (CC BY-NC-ND 4.0) License. 